\title{
Percolation and Deconfinement in SU(2) Gauge Theory
}

\author{
S. Fortunato ${ }^{\mathrm{a} *}$ and H. Satz \\ ${ }^{a}$ Fakultät für Physik, Universität Bielefeld, \\ D-33501 Bielefeld, Germany
}

The deconfinement transition in $\mathrm{SU}(2)$ gauge theory and the magnetization transition in the Ising model belong to the same universality class. The critical behaviour of the Ising model can be characterized either as spontaneous breaking of the $Z_{2}$ symmetry of spin states or as percolation of appropriately defined spin clusters. We show that deconfinement in $\mathrm{SU}(2)$ gauge theory can be specified as percolation of Polyakov loop clusters with Fortuin-Kasteleyn bond weights, leading to the same critical exponents as the conventional order-disorder description based on the Polykov loop expectation value.

\section{INTRODUCTION}

The study of critical phenomena has always been one of the most challenging and fascinating topics in physics. One can give many examples of systems which undergo phase transitions, from familiar cases like the boiling of water in a kettle or the paramagneticferromagnetic transition of iron at the Curie temperature, to the more complicated case of the transition from hadronic matter to quark-gluon plasma which is likely to be obtained by high-energy heavy-ion experiments in the coming years. Particularly interesting are the second-order phase transitions, characterized by a continuous variation of the order parameter and a divergent correlation length at the threshold. Already in the 70's one tried to use percolation theory in order to give a geometrical description of the critical behaviour of dynamical systems undergoing second-order phase transitions [1] 3]. Percolation has in fact several features in common with such systems: power law behaviour of the variables at criticality, scaling relations, universality.

The critical behaviour of the Ising model can indeed be reformulated in terms of percolation theory: magnetization sets in when suitably defined clusters of parallel spins reach the dimensions of the system [4]. In particular, the critical exponents for percolation then become equal to the Ising exponents.

The paramagnetic-ferromagnetic transition of the Ising model is strictly related to the confinement-deconfinement transition in finite temperature $S U(2)$ gauge theory. This was conjectured on the basis of effective theories [5,6] and confirmed by lattice studies [7]. The order parameter for $S U(2)$ is the lattice average of the Polyakov loop, and it behaves like the magnetization in the Ising model; in particular, the critical exponents are the same.

*The work has been supported by the TMR network ERBFMRX-CT-970122 and the DFG under grant Ka 1198/4-1. 
These analogies are the basis of this work. We have tried to see whether it is possible to find a description of critical behaviour in terms of percolation also for the deconfinement transition in $S U(2)$ gauge theory. We show that in a lattice regularization which corresponds to the strong coupling limit, both in two and in three space dimensions, the percolation of Polyakov loop clusters (taken to be suitably defined areas of Polyakov loops $L$ of the same sign) leads to the correct deconfinement temperature and to the correct critical exponents for the deconfinement. We have achieved this result by means of computer simulations of finite temperature $S U(2)$ gauge theory and using standard finite size scaling tecniques to extrapolate the results to the infinite volume limit.

\section{PERCOLATION THEORY AND THE ISING MODEL}

The percolation problem [8,9] is easy to formulate: just place randomly pawns on a chessboard. Regions of adjacent pawns form clusters. Percolation theory deals with the properties of these clusters when the chessboard is infinitely large. If one of the clusters spans the chessboard from one side to the opposite one, we say that the cluster percolates.

Quantitatively, one counts how many pawns belong to each cluster and calculates two quantities:

- The average cluster size $\mathrm{S}$, defined as:

$$
S=\sum_{s}\left(\frac{n_{s} s^{2}}{\sum_{s} n_{s} s}\right) .
$$

Here $n_{s}$ is the number of clusters of size $s$ and the sums exclude the percolating cluster; this number indicates how big on average the clusters are which do not percolate.

- The percolation strength $\mathrm{P}$, defined as:

$$
P=\frac{\text { size of the percolating cluster }}{\text { no. of lattice sites }} .
$$

By varying the density of our pawns, a kind of phase transition occurs. We pass from a phase of non-percolation to a phase in which one of the clusters percolates. The percolation strength $\mathrm{P}$ is the order parameter of this transition: it is zero in the nonpercolation phase and is different from zero in the percolation phase.

If we want to study percolation in dynamical systems, for example the Ising model, first of all we must define the rule to build up the clusters (for instance we can join together all nearest-neighbour spins of the same sign). Then one has to find out at which temperature we have a spanning cluster. Let us call this temperature $T_{p}$. It turns out that for $T \approx T_{p}$ the percolation variables $\mathrm{P}$ and $\mathrm{S}$ behave in the following way:

$$
P \sim\left(T_{p}-T\right)^{\beta_{p}}, \quad S \sim\left|T-T_{p}\right|^{-\gamma_{p}}
$$

A. Coniglio and W. Klein [1] demonstrated that for some special definition of cluster, the onset of percolation coincides with the one of magnetization; besides, $\beta_{p}$ and $\gamma_{p}$ coincide respectively with the magnetization exponent $\beta$ and the susceptibility exponent $\gamma$. Such cluster definition had already been used by Fortuin and Kasteleyn to show that the partition function of the Ising model can be rewritten in purely geometrical terms as a sum 
over clusters configurations [10]. According to the Fortuin-Kasteleyn prescription, two nearest-neighbouring spins of the same sign belong to the same cluster with a probability $p=1-\exp (-2 \beta)(\beta=J / k T, \mathrm{~J}$ is the Ising coupling, $\mathrm{T}$ the temperature). The result of Coniglio and Klein is valid in any space dimension $d(d \geq 2)$ and it is independent of the lattice geometry (square, triangular, honeycomb, etc.) as long as it is homogeneous.

\section{POLYAKOV LOOP PERCOLATION IN SU(2) GAUGE THEORY}

Finite temperature $S U(N)$ gauge theories describe the interactions of systems of mutually interacting gluons in thermal equilibrium ( $N$ is the number of colours). Systems containing quarks together with gluons are certainly more appealing; such a scenario will be explored experimentally up to very high temperatures by means of high-energy heavy ion collisions. Nevertheless, $S U(N)$ gauge theories are of theoretical interest; already at this level one has a transition between confinement to deconfinement, going from a phase of bound state of gluons (glueballs) to a phase of free gluons. The order parameter of the confinement-deconfinement phase transition of $S U(N)$ gauge theories is the lattice average of the Polyakov loop $\langle L>$. It is related to the effective potential $V(r)$ of a static (mass $\rightarrow \infty$ ) quark-antiquark pair put in the gluons' medium at temperature $T$ at a distance $r$ from each other, when $r$ is very big:

$$
|<L>|^{2} \sim \lim _{r \rightarrow \infty} e^{-\frac{V(r)}{T}}
$$

The Polyakov loop is zero in the confined phase $(V(r) \rightarrow \infty)$ and different from zero in the deconfined phase $(V(r)$ finite). Some time ago it was conjectured by B. Svetitsky and L. G. Yaffe that the critical behaviour of $S U(N)$ gauge theories has a strong relationship with the one of simple $Z(N)$ spin models, with which they share a common global symmetry [6]. In particular, in case of second order phase transitions, both models would belong to the same universality class, that is they would have the same set of critical exponents. One simple test of the Svetitsky-Yaffe conjecture is provided by the $S U(2)$ gauge theory: numerical simulations showed that its critical exponents indeed coincide with the ones of the Ising model [7].

In some respect the lattice configurations of $S U(2)$ are similar to the ones of the Ising model. Instead of having a spin variable at each lattice site we have the value of the Polyakov loop at that site. Our aim is to see whether it is possible to build clusters of Polyakov loops such that their percolation indices (threshold, exponents) coincide with the thermal ones. In order to do that, we have to face two difficulties:

i) the Polyakov loop is not a two-valued variable like the spin in the Ising model but a continuous one; its values vary in a range that, with the normalization convention we use, is $[-1,1]$;

ii) the $S U(2)$ Lagrangian is not directly a function of the Polyakov loop, therefore it is not possible to extract from it the expression of the bond probability that we need to build the clusters like in the Ising model.

In a recent work [11] it was proved that the point i) is not a problem. If we take an Ising model with continuous instead of two-valued spins, the definition of Fortuin-Kasteleyn clusters can be generalized by taking as a bond probability between two positive (or 
negative) nearest-neighbour spins $s_{i}$ and $s_{j}$ the following expression:

$$
p(i, j)=1-\exp \left(-2 \beta s_{i} s_{j}\right)
$$

The problem ii), however, is hard to overcome. In particular, it seems that there is no way to express the lattice Lagrangian of $S U(2)$ in terms of Polyakov loops for all lattice regularizations. Therefore we were forced to investigate $S U(2)$ in a special lattice regularization, which corresponds to the so called strong coupling limit. In this case it was shown [12 that the partition function of $S U(2)$ can be written in a form which, apart from a factor which depends on the group measure, is the partition function of the continuous Ising model studied in [11]. The coupling $\kappa$ of the spin model and the coupling $\beta$ of $S U(2)$ are related to each other by this relation:

$$
\kappa \simeq(\beta / 2)^{N_{t}}
$$

( $N_{t}$ fixes the temporal lattice regularization). If $S U(2)$ is approximately a continuous Ising model, we can try to use the general definition of clusters of [11]. Therefore we have defined our clusters as regions of like-sign Polyakov loops connected by bonds distributed according to the bond weight

$$
p(i, j)=1-\exp \left(-2 \kappa L_{i} L_{j}\right),
$$

( $L_{i}$ and $L_{j}$ are the Polyakov loop values at the sites $i$ and $j$ ). For $N_{t}=2$ the approximation of [12] is good and we chose to investigate numerically this special case in two and three space dimensions.

\section{RESULTS}

We began our percolation studies performing some test runs for different lattice sizes to check the behaviour of our percolation variables around criticality. Figure 1 shows the average cluster size $S$ for $2+1 S U(2)$ in correspondence of different lattice sizes. To get the critical point of the percolation transition we used the method suggested in [13]. For a given lattice size and a value of $\beta$ we counted how many times we found a percolating cluster. This number is successively divided by the total number of configurations at that $\beta$. We call this quantity percolation probability. This variable is directly a scaling function, analogue to the Binder cumulant in continuous thermal phase transitions. Figure 2 shows the percolation probability for the 3 -dimensional case as a function of $\beta$ for $24^{3} \times 2$, $30^{3} \times 2$ and $40^{3} \times 2$.

The lines cross at the same point within the errors and that restricts further our $\beta$ range for the critical threshold. Besides, since the percolation probability is a scaling function, we could already get clear indications about the class of critical exponents of our clusters. In fact, if one knows the critical point and the exponent $\nu$, a rescaling of the percolation probability as a function of $\left(\beta-\beta_{c r}\right) L^{1 / \nu}$ should give us the same function for each lattice size. Fig. 3 and 4 show the rescaled percolation probability for 3-dimensional $S U(2)$ using $\beta_{c r}=1.8747$ and two different values of the exponent $\nu$, respectively the Ising value and the random percolation one. The figures show clearly a remarkable scaling for the Ising exponent and no scaling for the random percolation exponent. 


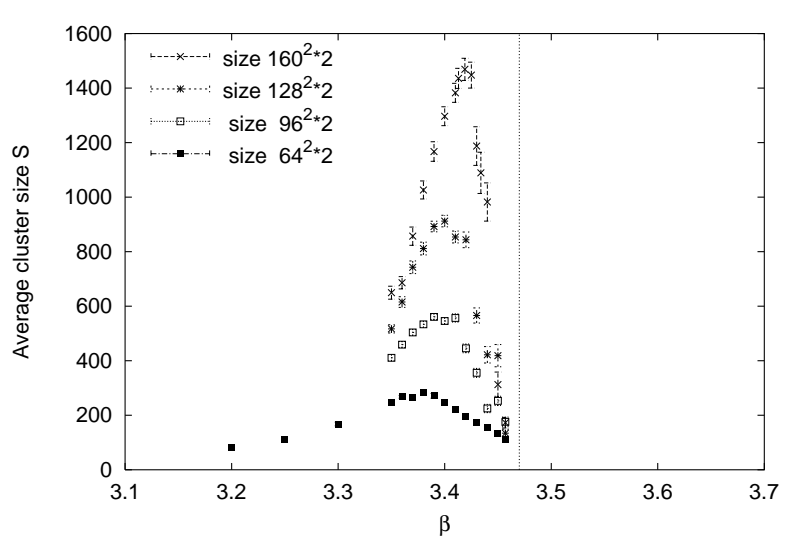

Figure 1. Average cluster size as a function of $\beta$ for $2+1 S U(2)$ and different lattice sizes. The dashed line indicates the position of the physical threshold.

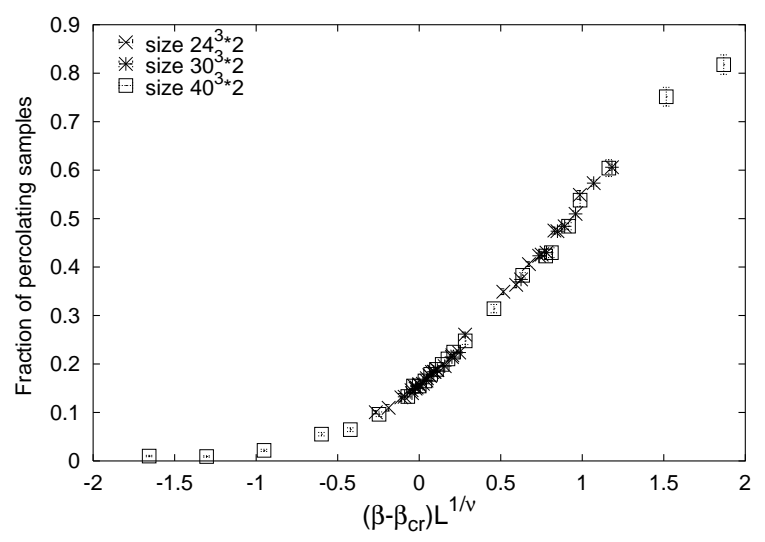

Figure 3. Rescaled percolation probability for $3+1 S U(2)$ using $\beta_{c r}=1.8747$ and the 3 -dimensional Ising exponent $\nu=0.629$.

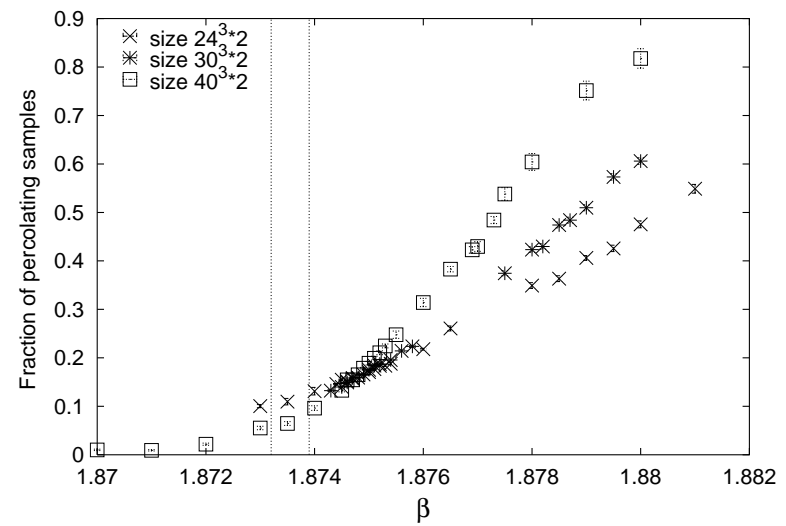

Figure 2. Percolation probability for $3+$ $1 S U(2)$ as a function of $\beta$ for three lattice sizes.

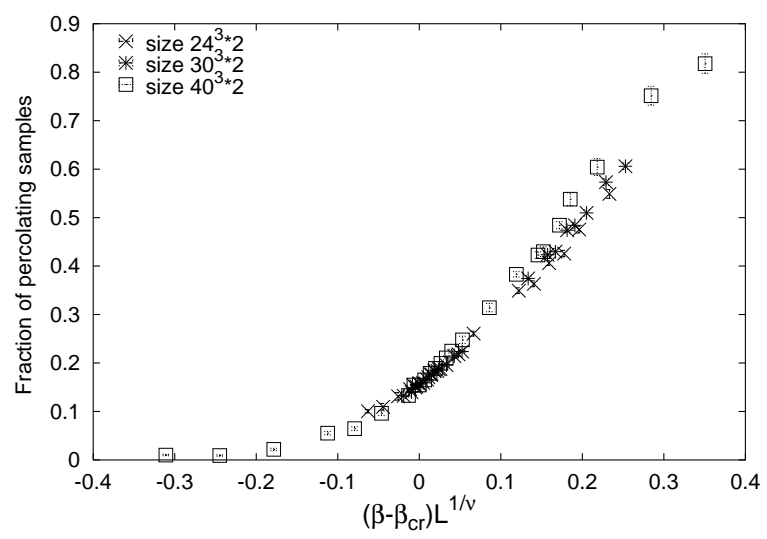

Figure 4. Rescaled percolation probability for $3+1 S U(2)$ using $\beta_{c r}=1.8747$ and the 3 -dimensional random percolation exponent $\nu=0.88$.

To evaluate the exponents $\beta$ and $\gamma$ we performed high-statistics simulations in the range where the percolation probability curves cross each other. The number of measurements we took for each value of the coupling varies from 50000 to 100000 . We used the $\chi^{2}$ method [7] to determine the values of the exponents. The final results are reported in Table 1 and 2. The agreement both in two and in three dimensions is good.

\section{CONCLUSIONS}

We have shown that the confinement-deconfinement phase transition in finite temperature $S U(2)$ pure gauge theory can be described as percolation of some suitably defined clusters of Polyakov loops of the same sign. Our result is valid only in the strong coupling limit, and in order to make it general a different approach seems to be necessary. The use 
Table 1

Comparison of the thermal and the percolation results for $2+1 \mathrm{SU}(2)$.

\begin{tabular}{lllll}
\hline & Critical point & \multicolumn{1}{c}{$\beta / \nu$} & \multicolumn{1}{c}{$\gamma / \nu$} & $\nu$ \\
\hline L Percolation & $3.443(1)$ & $0.128(5)$ & $1.752(8)$ & $0.98(5)$ \\
Spont. Symm. Breaking & $3.464(14)$ & $1 / 8$ & $7 / 4$ & 1 \\
\hline
\end{tabular}

Table 2

Comparison of the thermal and the percolation results for $3+1 \mathrm{SU}(2)$.

\begin{tabular}{lllll}
\hline & Critical point & $\beta / \nu$ & $\gamma / \nu$ & $\nu$ \\
\hline L Percolation & $1.8747(2)$ & $0.528(15)$ & $1.985(13)$ & $0.632(11)$ \\
Spont. Symm. Breaking & $1.8735(4)$ & $0.523(12)$ & $1.953(18)$ & $0.630(14)$ \\
Ising Model [14] & & $0.518(7)$ & $1.970(11)$ & $0.6289(8)$ \\
\hline
\end{tabular}

of effective theories for $S U(2)$ may help to solve the problem [15.

\section{REFERENCES}

1. H. Müller-Krumbhaar, Phys. Lett. 48 A , 459 (1974).

2. M. F. Sykes, D. S. Gaunt, J. Phys. A: Math. Gen. 9, 2131-2137 (1976).

3. A. Coniglio, C. Nappi, F. Perrugi, L. Russo, J. Physics A: Math. Gen. 10 205-218 (1977)

4. A. Coniglio, W. Klein, J.Phys. A: Math. Gen. 13, 2775-2780 (1980).

5. J. Polónyi, K. Szlachányi, Phys. Lett. B 110, 395 (1982).

6. B. Svetitsky, L. G. Yaffe, Nucl. Phys. B 210 [FS6] 423 (1982).

7. J. Engels et al., Phys. Lett. B 365219 (1996).

8. D. Stauffer, A. Aharony, Introduction to Percolation Theory, Taylor \& Francis, London 1994.

9. G. Grimmett, Percolation, Springer, 1999.

10. C. M. Fortuin, P. W. Kasteleyn, Physica 57536 (1972).

11. P. Bialas, P. Blanchard, S. Fortunato, D. Gandolfo, H. Satz, hep-lat/9911020, Nucl. Phys. B (in press)

12. F. Green, F. Karsch, Nucl. Phys. B 238, 297 (1984).

13. K. Binder, D. W. Heermann, Monte Carlo Simulations in Statistical Physics, An Introduction, Springer-Verlag 1988, 40-41

14. A. M. Ferrenberg, D. P. Landau, Phys. Rev. B 445081 (1991).

15. S. Fortunato, F. Karsch, P. Petretski, H. Satz, in progress. 